\title{
Highlights of the 11th Emirates Diabetes and Endocrine (Virtual) Congress, March 4-6, 2021
}

\author{
Salem A. Beshyah ${ }^{\mathrm{a}}$ Fauzia Rashid $^{\mathrm{b}}$ Elamin Ibrahim Abdelgadir ${ }^{\mathrm{b}}$ \\ aDepartment of Medicine, Dubai Medical College for Girls, Dubai, United Arab Emirates; \\ ${ }^{b}$ Endocrinology Department, Dubai Hospital, Dubai, United Arab Emirates
}

\section{Keywords}

Diabetes · Thyroid · Pituitary · Metabolism · Adrenal · Lipids . Dubai $\cdot$ UAE $\cdot$ Hypogonadism · Polycystic ovary · Growth . COVID-19· Education · Research · Obesity

\begin{abstract}
Backgrounds: The Eleventh Diabetes and Endocrine Virtual Congress (EDEC 2021) was held on March 4-6, 2021, due to the COVID pandemic. Objectives: We aimed to present highlights of the congress proceedings. Materials and Methods: The journal appointed 3 rapporteurs to prepare this congress report. They prepared assigned sections and reviewed the rest of the manuscript, and approved its final version. Results: The conference contents over 3 days included a wide range of lectures by world-class experts and key opinion leaders on various topical subjects, endocrinology, diabetes care, and metabolism, in addition to original data from submitted abstracts. The clinical approach to managing a pituitary mass, contemporary management of acromegaly, and the relationship between growth hormone and neoplasia were discussed. The virtual EDEC 2021 provided a comprehensive review of topical issues concerning clinical practice and research in diabetes, endocrinology, and metabo-
\end{abstract}

lism. Predictably, the thyroid took the scene's center, spanning many subjects from hypothyroidism, pregnancy care, thyroid nodules, and cancer management. Rational approaches to pituitary disease and acromegaly were valuable for practicing endocrinologists. Recent advances in adrenal disease were valuable, practical, and futuristic. The reviews on bone metabolism and calcium homeostasis at large and during the COVID-19 pandemic were fascinating. On the diabetes side, emphasis on the cardiovascular outcomes, the use of newer agents when compelling indications exist, and the role of technology were precious contributions. Some of the classical questions were addressed and revisited, such as endocrinology of thalassemia, precocious puberty, and testosterone replacement. The newer and future lipid-modifying therapies were stimulating, mainly when high-risk patients are considered. Imaging, immunology, and molecular biology took their share in various presentations. Conclusions: The virtual EDEC 2021 provided a comprehensive review of topical issues concerning clinical practice and research in diabetes, endocrinology, and metabolism. Many new concepts were introduced in diabetes care and endocrinology, bringing the audience to the forefront of research and world-class clinical practice.

(c) 2022 The Author(s)

Published by S. Karger AG, Basel karger@karger.com www.karger.com/dde

Karger $\stackrel{\text { ' }}{5}$

BOPEN ACCESS
(C) 2022 The Author(s)

Published by S. Karger AG, Basel

This is an Open Access article licensed under the Creative Commons Attribution-NonCommercial-4.0 International License (CC BY-NC) (http://www.karger.com/Services/OpenAccessLicense), applicable to the online version of the article only. Usage and distribution for commercial purposes requires written permission.
Correspondence to:

Salem A. Beshyah, beshyah@yahoo.com 


\section{Introduction}

The Emirates Diabetes and Endocrinology Congress (EDEC) first took place in 2010 and has established its position over the last decade as a scientifically enriched event targeting the educational demands of diabetologists, endocrinologists, and all other professions with a particular interest or clinical responsibilities in these fields.

The EDEC used to be held physically in Dubai since its inception. However, the Eleventh Diabetes and Endocrine Virtual Congress (EDEC 2021) was held online on March 4-6, 2021, as a virtual event. However, this allowed a broad choice of speakers worldwide to participate without being restricted by time used for distant travel. The primary aim of the congress has always been to deliver an update of the state of the art in clinical practice and research in diabetes, endocrinology, and metabolism similar to its predessors [1].

\section{Materials and Methods}

Three rapporteurs were commissioned to prepare this report. They prepared assigned sections, reviewed the rest of the manuscript, and developed the whole report. The aim was to present highlights of the congress proceedings to extend the benefit to those who could not attend the meeting live. Targeted readership includes established physicians, doctors in training, specialist nurses, and educators interested in diabetes, endocrinology, and metabolism. The abstracts of the free communications were published separately [2].

\section{Results: Congress Highlights}

The congress included several "state-of-the-art" lectures (Table 1) and clinical management symposia and met the expert sessions. Up to 4 virtual halls were open at various times to accommodate different options to meet the delegates' choices within 3 days of the congress. Contents of the scientific program included endocrine tracks (Table 2), diabetes care tracks (Table 3), and a miscellaneous track of free communications, pediatrics, $\mathrm{CO}$ VID-19, and diabetes educators' session (Table 4). Speakers were invited from renowned national, regional, and international key opinion leaders. Numerous industrysponsored sessions occurred during the event covering newer pharmaceutical agents, trials, and recent conceptual developments. However, this report will not be included since it was organized independently from the sci-
Table 1. State-of-the-art lectures

Diabetes prevention and CVD

Bariatric surgery: devil or divine

NET imaging

Thyroid and pregnancy

Immunotherapy in type 1 diabetes - Can we prevent type 1

diabetes?

Is GH implicated in cancer?

Immunotherapy-induced endocrine disease

Diabetes data science: the VIP approach to personalized diabetes

medicine

Monogenic forms of diabetes

NET, neuroendocrine tumor; GH, growth hormone.

entific committee. The contents of the congress will be highlighted conceptually, and complex numerical details will be avoided. They are presented under 3 subheadings of endocrinology, diabetes, and metabolism.

\section{Endocrinology}

\section{Pituitary}

Clinical Approach to Managing a Pituitary Mass

Professor Shlomo Melmed (USA) gave an extensively illustrated presentation on the clinical approach to managing pituitary masses. He covered a wide range of tumor size and function. He discussed the best practice of managing pituitary masses in adults from incidentalomas to giant and aggressive pituitary tumors. Perhaps the most important initial question is whether the patient requires surgery, such as pressure effects, nonprolactinomas, and increased size? He described the subclass of silent tumors that may be positive on staining and are more likely to recur. He summarized a paradigm for nonsecretory pituitary tumors, which considers (1) making a differential diagnosis by clinical features and MRI scan and (2) performing dynamic endocrine pituitary reserve tests and recognizing nonfunctioning pituitary tumors (macro- and microadenomas) and nonpituitary sellar masses. He recommended first excluding an aneurysm for nonpituitary sellar masses and proceeded with surgery and established a histological diagnosis to treat accordingly. However, for nonfunctioning pituitary adenomas, he suggested MRI monitoring for macroadenomas and pituitary secretary reserve. On the other hand, for macroadenomas, he recommended considering the need for surgery, histology-based disease-specific therapy in addition to MRI monitoring, and trophic hormone assessment and replacement. 
Table 2. Endocrinology contents of the scientific program

Risk stratification of thyroid nodules

Monitoring strategies for patients with DTC

The utility and complexity of diagnostic molecular analysis in thyroid nodule Care Overview of syndromes of resistance to thyroid hormone

Treating hypothyroidism in 2021: Is it that simple?

Clinical approach to managing the pituitary mass

Acromegaly treatment updates

A simplified approach to primary aldosteronism and endocrine hypertension

New steroids and new treatments for $\mathrm{CAH}$

Practical approach to osteoporosis

New insights into hypoparathyroidism

Guideline-based management of testosterone deficiency syndromes in men

Monogenetic obesity and new treatments

Adolescent endocrinology

Advances in precocious puberty

Management of endocrinopathies in patients with thalassemia

$\mathrm{CAH}$, congenital adrenal hyperplasia; DTC, differentiated thyroid cancer.

Table 3. Diabetes and metabolism (including obesity and lipids) contents of the scientific program

What is on the horizon for diabetes management

Use of devices in people with diabetes

A century of diabetes technology: signals, models, and artificial pancreas control

TIR versus $\mathrm{HbA} 1 \mathrm{c}$

Management of diabetic foot: how are we doing regionally?

Diagnosis and treatment of DSPN in clinical practice

Counseling on the fundamentals of lifestyle change for obesity: diet, physical activity, and behavior

Update on present and future LMT

Pharmacotherapy for obesity - understanding the appetite-behavior connection

$\mathrm{NASH}$

TIR, time in range; DSPN, diabetic sensorimotor polyneuropathy; LMT, lipid-modifying therapy.

Table 4. Special sessions

1. Oral Free communications* [selected from submitted abstracts]

Epidemiological aspects of Adrenal Insufficiency Detected by the Synacthen Test and Insulin Hypoglycemia During Withdrawal from Prolonged Corticosteroid Therapy

Vitamin A Levels Are Reduced in Diabetic-Emirati Subjects and Not Influenced by Antidiabetic Medications

The alpha-subunit of glycoprotein hormones and anatomopathological aspects of pituitary adenomas

Metabolic Surgery Effects on Patients with and without Type-2 Diabetes: An Analysis of Primary Roux-en-Y Gastric Bypass and Sleeve

Gastrectomy Outcomes

2. Diabetes and endocrine data from UAE*

Incidence and risk factors gestational diabetes mellitus in UAE: The Mutaba'ah study

Complications associated with type 1 diabetes: Can genetic studies tell us more?

Control of cardio-metabolic risk parameters

3. Diabetes and endocrine challenges in the COVID-19 pandemic

Two presentations impact of COVID-19 pandemic on (1) diabetes and (2) bone health and calcium homeostasis

4. Diabetes educators session

Carbohydrate counting for effective insulin dosing in T1D

Continuous glucose monitoring systems (GCMS)

* No further details are provided here since these were not fully published after peer review. All free communications are published elsewhere in this journal [2]. 
Acromegaly Treatment Updates

Acromegaly is a syndrome with excess growth hormone $(\mathrm{GH})$ production. Its features result from either the direct effect of $\mathrm{GH}$ or inducing overproduction of the insulin-like growth factor-1 (IGF-1). He reminded the audience of the physical and metabolic features of acromegaly. Surgical treatment is the standard initial treatment option, followed by medical treatment. Some patients may become more resistant to medical treatment than others; this could be attributed to the granulation of the pituitary adenoma (densely granulated responds better to medical treatment). At the same time, the sparsely granulated are more prone to recur or to become.

Moreover, specific receptor expression on the surface of tumor cells, either as a somatostatin receptor or as a dopamine receptor, may allow a personalized approach or a marker of somatostatin responsiveness. The patient's age is inversely proportional to the potential therapeutic outcome, in that younger patients exhibit far more adverse outcomes than older patients. The cell cycle markers like KO67 and P21 can also help decide on the aggressiveness of the tumor.

The speaker showed data on octreotide-resistant tumors and the effect of pasireotide in this group of patients. He eluded to the side effects of the pasireotide compared to the octreotide, which is relatively the same, including gallbladder stones, hyperglycemia, diarrhea, and injection site discomfort. Prof. Melmed also showed extensive data on the combination of somatostatin analogs and pegvisomant. The combination therapies have shown a much higher rate of postoperative IGF-a control, fewer side effects, and less annual medication spending.

\section{Is $\mathrm{GH}$ Implicated in Cancer?}

Professor Shlomo Melmed from the USA discussed a novel topic for the potential role of GH in cancer. Conventionally, GH is known for its action on skeletal growth. However, further studies about the hypothalamic-GHIGF axis yield that GH works in a far more complex manner in the peripheral system through the dimeric receptors and bring many cellular and metabolic changes in the liver, pancreas, muscles, kidneys, colon, adipose tissues, bone, and heart. Dr. Shlomo Melmed used the colon as a model to discuss the GH-IGF axis role for the implication of cancer. Through its dimeric receptor, GH induces multiple cascades of intracellular signaling and results in the production of IGF-1, cell proliferation gene, glucose metabolism, nuclear pre-proliferation gene, $\mathrm{C}$-fos, etc.

The effects of the pre-proliferation gene on multiple soft tissue proliferation are well known, e.g., goiter, ton- sillar hypertrophy, and cardiac muscle hypertrophy. The presence of megacolon, lipoma, and fibroma is pathognomonic for high GH levels. Whether the same molecular changes can also cause cancer in cases of high GH was elaborated. A few studies from Italy and Argentina showed that thyroid, kidney, and colorectal malignancies are significant in acromegaly patients. It is not related to IGF level, radiotherapy, and disease duration, except the only correlation, was a positive family history of malignancy. Contrary to this, a German registry did not find any association of malignancy with acromegaly.

Dr. Melmed concluded that the current literature is not very clear about the hypothesis of GH's role in the implication of cancer. However, GH acts as an endocrine, paracrine, autocrine, or intracrine hormone, and the intracellular level of GH correlates with cancer. He proved this hypothesis by presenting many preclinical and clinical studies that although GH is required for growth, homeostasis, and maintenance in different life stages, the high intracellular GH suppresses the P53 gene that eludes proliferation arrest. It enables a pro-proliferative mucosal field change and prevents DNA damage repair. Vice versa, low GH entropy enriches familial longevity.

\section{Thyroid}

Treating Hypothyroidism in 2021: Is It that Simple?

Bryan Haugen presented the data that compared thyroid symptoms scores from people with normal thyroid function versus those with hypothyroidism. He discussed a prevalent yet complex issue of those patients on levothyroxine with biochemical euthyroidism but persistent hypothyroid symptoms and supported the use of LT4 in these cases after excluding other causes for their symptoms. However, he treats by combining T4/T3 (10-14:1 ratio) only those that do not clinically well despite being on target TSH.

\section{Thyroid and Pregnancy}

Erik Alexander (USA) discussed the association of various spectrums of maternal thyroid disorder with risk to fetus and pregnancy. He discussed the most recent highquality data regarding the treatment of mild hypothyroidism in early pregnancy and treatment questions in TPO-positive women before pregnancy with different scenarios. The recent randomized prospective controlled trial data suggest no benefit of treatment in the setting of mild subclinical hypothyroidism in early gestation for fetal loss, preterm delivery, or IQ benefit in offspring. However, there are also existing studies that have shown some 
variable harmful effects of untreated mild hypothyroidism. Dr. Alexander translated this variation for the 2021 standard and suggested a TSH of at least $4.0 \mathrm{mIU} / \mathrm{L}$ to define hypothyroid. Due to the substantial risk of miscarriage, earlier treatment before 12 weeks of gestation seems to offer a proven benefit in this regard. There is no value of treatment by thyroxine before pregnancy in euthyroid women, irrespective of their anti-TPO status and titer. However, it is impactful once pregnant and needs treatment for patients with positive antibodies and TSH of $>2.5 \mathrm{mIU} / \mathrm{L}$.

\section{Risk Stratification of Thyroid Nodules}

Dr. Sarah Mayson addressed the advantages and limitations of the commonly used scoring systems in risk stratification of the thyroid nodules. She discussed the rationale for evaluating thyroid nodules based on ultrasound features/patterns. The composition, echogenicity, margins, echogenic foci, shape, and vascularity of the nodules are primarily taken into account. Dr. Mayson briefly discussed commonly used ultrasound risk stratification systems (RSS) from 2009 to till 2017 from various organizations of different countries. The most utilized are ATA management guidelines for adult patients with thyroid nodules and differentiated thyroid cancer (DTC) (2015), with the principle that benign or suspicious features tend to occur together. Use pattern recognition to determine malignancy risk and the need for FNA. The other commonly used system in the USA is thyroid ultrasound reporting lexicon: white paper of the ACR Thyroid Imaging, Reporting and Data System (ACR TI-RADS) Committee, with the principal to determine the point value for each architectural category, total the points, and determine the TI-RADS level for the need of FNA with the following categories: TR0-2 no FNA, TR3 FNA if $>2.5 \mathrm{~cm}$, follow if $>1.5 \mathrm{~cm}$, TR4, FNA $>1.5 \mathrm{~cm}$, follow if $>1 \mathrm{~cm}$, TR5 FNA $>1 \mathrm{~cm}$, follow if $>0.5 \mathrm{~cm}$. She also presented many studies and metaanalyses to compare different RSS for their relative ability to select malignant nodules for FNA. Dr. Mayson concluded that most studies evaluating RSS offer lowquality evidence, and there are significant differences in the number of avoiding FNA across the system. The utilization of the ultrasound risk stratifications system improves our ability to identify benign and malignant nodules. RSS can be used to rule out thyroid malignancy, while FNA and cytology are still needed to confirm the diagnosis. More robust research is required for MTC and follicular thyroid cancers and also to validate existing RSS.
The Utility and Complexity of Diagnostic Molecular

Analysis in Thyroid Nodule Care

Dr. Erik Alexander had a very informative lecture about The Utility and Complexity of Diagnostic Molecular Analysis in thyroid nodule care over the last decade. He discussed various molecular analysis methods and the fact that we do not directly compare them due to the lack of high-quality validation studies. These diagnostic molecular analyses are used for diagnosis and improve care in these cases of an indeterminate and nondiagnostic thyroid nodule. He discussed the growing enthusiasm in RNA gene sequencing classifiers due to being more potent in molecular diagnostic and prognostic testing, and the results are binary benign versus suspicious. The DNAbased test looks for mutations and rearrangements. Gene expression appears superior to mutation analysis due to many supportive reasons in different studies, along with the fact that detection of a mutation/translocations does not always mean a malignancy. Dr. Alexander also described the recent advances in the molecular analysis of unique Hurthle cell neoplasm and the availability of these 2 test analyzers, i.e., Afirma GSC and Thyroseq v3, which use mRNA mtRNA expression analysis with loss of heterozygosity in such cases. Molecular data are robust as a prognostic tool, and BRAF-like, RAS-like, and NBNRlike testing are already into clinical practice for independent prognostic grouping.

\section{Monitoring Strategies for DTC}

Dr. Haugen discussed staging, risk of recurrence, response to therapy, and time since last therapy to efficiently use strategies for long-term monitoring in patients with DTC. For monitoring, he discussed the role and caveats of various monitoring tools such as the level of serum thyroglobulin ( $\mathrm{Tg}$ ) as a tumor marker, anatomic imaging by neck $\mathrm{U} / \mathrm{S}, \mathrm{CT}, \mathrm{MRI}$, and imaging like radioiodine (RAI) and FDG-PET and other nucleotide imaging for functional nature of DTC. The RAI whole-body scan identifies disease for therapeutic target, and the FDG-PET scan is meaningful for Tg-imaging mismatch, for new disease discovery and prognosis by several FGD avid lesions. Dr. Haugen specifically endorsed the practice of preoperative measurement of serum $\mathrm{Tg}$ and $\mathrm{TgAb}$ to assess the interfering effect of $\mathrm{Tg} \mathrm{Abs}$ on the Tg level in all these patients for the reliability of $\mathrm{Tg}$ to use as a postoperative tumor marker.

\section{Overview of Syndromes of Resistance to Thyroid} Hormone

Dr. Carla Moran (Ireland) presented an in-depth review of thyroid hormone resistance (THR) and its ge- 
nomic pathway of action via thyroid hormone receptors $\alpha$ and $\beta$ (RTH $\alpha$ and RTH $\beta$ ) that are differentially expressed in various body organs. THR occurs at various levels in the activation pathway of $\mathrm{TH}$; defective $\mathrm{TH}$ signaling through RTH $\beta$ and RTH $\alpha$; defective TH transport (MCT8 defects); and defective TH metabolism (SBP2 deficiency). Resistance due to TH $\beta$ signaling has very typical biochemistry of having an invariably raised FT4, variably high FT3, and normal TSH value due to THR at the level of hypothalamic-pituitary-thyroid axis. Due to the resistance of $\mathrm{TH}$ at the hepatic level in the $\mathrm{TH} \beta$ cases, patients have low SHBG levels, high total cholesterol, LDL, triglyceride levels, and low HDL level. The MRI spectroscopy showed high ectopic fat and elevated HOMA-IR. All these features are reminiscent of metabolic syndrome and suggestive of high insulin resistance and at risk of developing diabetes and cardiovascular disease later. Features known to be associated with RTH $\beta$ include goiter, nonraised SGBG, dyslipidemia, raised HOMA-IR, raised metabolic muscle rate, failure to thrive in childhood, ADHD, poor attention, reduced IQ, anxiety, low BMD, short stature, palpitations, arrhythmias, and cardiac failure. Most patients require no specific treatment. The treatment option for RTH $\beta$ is usually by beta-blockers for most patients for CV effects. The use of carbimazole is not recommended as it may result in pituitary hyperplasia and goiter formation. TRIAC* (tri-iodothyroacetic acid) is a naturally occurring thyroid hormone analog, and it works on pituitary thyrotropin. It is shown to act preferentially on the $\beta$ form of thyroid hormone receptors and thus helps to lower the TSH and lower the FT3 and FT4. In RTH $\alpha$, the organs are affected by muscle, bone, CNS, and heart due to predominant alpha receptors in these tissues. The clinical manifestations include low metabolic rate, obesity, development delay, dyspraxia, reduced IQ, seizure, dysarthria, skeletal dysplasia, high $B M D$, increased head circumference, anemia, constipation, the typical facial feature of the broad face, broad nasal bridge, puffy lips, and macroglossia. Biochemically, these patients have low or normal thyroid function tests, low T4/T3 ratio, low reverse T3, normal/high TSH, and $\mathrm{CK}$ high/normal as they have normal $\beta$ form of receptors. Most of them respond to thyroxine. The defective TH transport due to MCT8 defects causes specifically decreased transport of thyroid hormone into the brain. These patients have central hypothyroidism with consequent neurocognitive impairment and peripheral thyrotoxicosis and typical effects, like tachycardia, increased metabolism, increased GFR, and low body weight. The biochemistry profile in this category is low FT4, normal or slightly elevated TSH, and invariably high FT3. Treatment is via TRIAC, with the primary endpoint being a change in the FT3 level. Lastly, he discussed various defects in thyroid hormone metabolism, i.e., different kinds of selenoprotein deficiencies with resultant multisystem disorder and different phenotypic manifestations in such cases.

\section{Bone Health and Calcium Homeostasis}

A Practical Approach to Osteoporosis

Dr. Clifford Rosen presented a wide range of latest data on osteoporosis management and the pros and cons of its therapeutic agents and their comparative effectiveness. He discussed that using a high dose of vitamin D played no role in preventing bone loss, and it is a supportive measure along with calcium. Members of the bisphosphonate group, such as alendronate, preserve bone density if used for at least 3 years. Bisphosphonates are found to be associated with atypical subtrochanteric fractures. Denosumab is another group used for osteoporosis therapy for the last 10 years and binds to RANKL to block the production of osteoclasts. It reduces the incidences of new vertebral fractures and $20 \%$ reduction in nonvertebral fractures compared to placebo, but the therapy needs to continue for its benefits as discontinuation causes new fractures. Antiresorptive therapy is an appealing choice for its immediate effect on building bone mass and is indicated as the first line of therapy for severe osteoporosis. So far, safety data with parathyroid hormone (PTH) analog are also outstanding, but the main caveat is the daily parenteral administration and high cost. They reduce $65 \%$ of new vertebral fractures and $53 \%$ of nonvertebral fractures in postmenopausal women. Abaloparatide showed a superior efficacy than teriparatide in increasing BMD of femur neck and lumbar spine and reducing the major osteoporotic fracture risk. Dr. Rosen also highlighted the role of Romosozumab (monoclonal antibodies to Sclerostin) in osteoporosis treatment. It induces marked increases in the femoral neck and spine bone density and reduces bone resorption within 12 months, given as within 12 months, and given as $210 \mathrm{mg}$ monthly injection for 12 months. It reduces vertebral fractures dramatically. There are reports of osteonecrosis of the jaw and a slight increase in ischemic cardiac events.

\section{New Insight into Hypoparathyroidism}

Prof. Clifford Rosen has presented one of the classical lectures, hypoparathyroidism, but he focused on the role of exercise intolerance as a quality-of-life issue in hypoparathyroidism patients. He started by reminding the 
common features of hypoparathyroidism and not forgetting the uncommon features, including laryngeal spasms, cardiomyopathy, and Parkinson-like syndromes. He pointed out that despite the major effect of PTH in bone metabolism and osteoclast/osteoblast stability, bone densitometry shows normal or increased density in those patients; however, the risk of fracture is an area of debate from different data cohorts. Insights from experimental studies to the clinical demonstration of the effect of PTH on fat metabolism systemically and in the bone marrow, specifically, have been elaborated. The speaker showed an image of a bone marrow biopsy of a 12-year-old girl with hypoparathyroidism. The bone marrow was occupied with adipose tissues replacing the normal bone marrow trabeculae. The image mimicked an osteoporotic bone biopsy. In experimental studies, PTH has been shown to induce lipolysis and increase fatty acid levels, which in turn help in the differentiation of the osteoblasts from stem cells and bone buildup. In hypoparathyroid patients, bone mineralization and bone turnover are both progressively impaired. Clinically, PTH replacement has reduced the fat volume in the bone marrow and increased the pace of healthy bone formation.

\section{Adrenals}

A Simplified Approach to Primary Aldosteronism

Dr. Richard Auchus (USA) delivered 2 presentations on adrenal disorders. The first was presented as a simplified approach to primary aldosteronism and endocrine hypertension. The points are: (1) excess renin-independent aldosterone, (2) most common cause of secondary hypertension, (3) there is high end-organ damage, (4) curable or targeted therapy is rational and practical, and (4) surprisingly low rates of screening and diagnosis $(<1 \%$ are ever screening perhaps partly due to fear of embarking and confusion of approach). It is a syndrome with many subtypes. However, there is a continuum with low renin hypertension. He argued strongly against the need to stop antihypertensive drugs from doing the screening test (aldosterone-to-renin ratio). Most drugs are OK for the screening test since most drugs raise both aldosterones, and hence if PRA is suppressed, the screening test is valid. Up to 4 weeks are needed off spironolactone and eplerenone but still OK if PRA is suppressed. Perhaps, best drugs are alpha1-blockers and verapamil. However, they can always rescreen after off drugs. He presented a complex algorithm that takes the patients through screening, confirmation, localization, and management choice. He gave a few pearls to using mineral receptor antagonists. These were: (1) start low and go slow, (2) spirono- lactone can be started as low as $12.5 \mathrm{mg} / \mathrm{day}$, (3) goal: normal blood pressure, potassium, and nonsuppressed PRA, (4) consider stopping other medications, (5) can add thiazide, loop diuretic for high $\mathrm{K} 5$ and expect some rise in serum creatinine (may need to stop or reduce the dose). Moreover, (6) finally switch to Eplerenone if there are side effects.

He ended up by highlighting where progress is needed: (1) getting more patients screened, (2) simplification of confirmatory testing, (3) other ways are needed to identify who has bilateral disease and does not need CT and AVS, (4) secondary criteria for AVS and predicting response to adrenalectomy, (5) dealing with the heterogeneity of primary aldosteronism, and (6) how best to titrate medical therapy.

New Steroids and New Treatments for CAH

The second presentation by Dr. Auchus addressed the recent advances in new steroids and treatment of 21 hydroxylase deficiency. His presentation was vibrant with the physiological and biochemical basis for the androgen excess and variable glucocorticoid and mineralocorticoid deficiency characteristic of congenital adrenal hyperplasia (CAH). He illustrated the diverted pathways both upand downstream. He used biomarkers of adrenal size in $21 \mathrm{OHD}$. He also illustrated the value of the 11 ketotestosterone-to-testosterone ratio. He also demonstrated the changes in several markers in children and adolescents and the changes in postmenopausal women. Regarding newer therapies for CAH, Dr. Auchus discussed the potential roles and supporting data for modified-release hydrocortisone, hydrocortisone subcutaneous infusion pump, and super androgen receptor antagonists including P450 17 A1 inhibitor (Abiraterone acetate), CRH receptor antagonists (NBI-77860, Tildacerfont, and Crinecerfront), and ACAT1 (SOAT1) inhibitor (Nevanimibe).

In summary, the news in CAH is twofold. Concerning newer androgens, 11-oxy androgens are dominant, and 21-deoxycortisol is a more specific marker. Experimental therapies include modified hydrocortisone delivery options, P450 17A1 inhibitor, CRFR1 antagonists, and SOAT1 inhibitor.

\section{Pediatric Endocrinology}

Advances in Precocious Puberty

Puberty entails several landmark changes in the life of adolescent males and females. These include: (a) the appearance and development of secondary sexual characteristics, (b) growth spurt and termination of somatic 
growth, (c) acquisition of reproductive capacity, and (d) psychological maturation. Jesus Argente (Spain) gave a comprehensive overview of precocious puberty. He started by reviewing the definitions and various events of puberty in males and females. Following this, he described the ages defining normal puberty, precocious, and delayed puberty. He described the various neuroendocrine and metabolic regulatory mechanisms. He acknowledged the contributions of several workers worldwide to the understanding of the triggers of the various events. He illustrated the sequence of pubertal events in boys and girls.

Central precocious puberty is caused by premature activation of the hypothalamic pulsatile production/secretion of gonadotropin-releasing hormone. The physiological mechanism of puberty onset is only partially understood but is believed to involve a shift in the balance of inhibitory, permissive, and excitatory hypothalamic factors on the gonadotropin-releasing hormone neurons. Several factors have a role in pubertal development, including metabolic, environmental, and genetic aspects. He elaborated on several examples of these.

He concluded by stressing a few crucial points: (a) although multiple advances in our knowledge of the onset of puberty have been made, its mechanisms remain partially understood, (b) MKRN3 mutations represent the most common monogenic cause of central precocious puberty, (c) DLK1 mutations give new insight into the correlation between obesity/metabolic syndrome and pubertal development, and finally (d) more research in new genes potentially involved in the etiology of CPP is necessary.

Management of Endocrinopathies in Thalassemia

Dr. Ramlah Al-Saif (KSA) started with a short introduction to the epidemiology of thalassemia and a short overview of iron metabolism. He discussed factors for endocrinopathies in thalassemia. He described the various endocrine disorders in thalassemia patients and reviewed the I-CAT guidelines and recommendations.

Endocrine dysfunction has been reported as the most common and earliest organ toxicity in thalassemia patients. A range of endocrine complications has been reported. Several studies demonstrated that $88 \%$ of thalassemia patients have at least 1 endocrine disorder. These include hypogonadism (60-70\%), growth failure (30$70 \%)$, diabetes (5-24\%), hypothyroidism $(8.7 \%)$, hypoparathyroidism (2\%), osteoporosis and osteopenia and adrenal insufficiency (0.7\%), and vitamin D deficiency.

To close, the speaker stressed that: (1) endocrine complications still occur despite advancement in the manage- ment of thalassemia patients, (2) teamwork should be an essential feature in patients' management, (3) optimum chelation therapy and strict compliance are crucial to minimizing complications, (4) regular screening for potential complications from 9 to 10 years after diagnosis, and finally (5) I-CET guidelines should be followed but may be modified according to patients' characteristics in each country.

\section{Management of Testosterone Deficiency in Men}

Prof. Shalender Bhasin addressed the difficulties in diagnosing testosterone deficiency and androgen deficien$c y$ in the elderly and the patient-centric management of diagnosis and testosterone replacement. He started with the diagnosis of androgen deficiency. He highlighted that the diagnosis of testosterone deficiency should be in 2 steps: clinical suspicion of hypogonadism, in addition to the exclusion of systemic causes such as cancer, HIV infection, other major chronic illnesses, or a variety of drugs or lifestyles that can be associated with testosterone deficiency. The second step is to measure fasting early morning total testosterone concentration using reliable and accurate assay such as liquid chromatography-tandem mass spectrometry-based assays. The low testosterone needs to be confirmed twice. Free testosterone only needs to be measured when the sex hormone-binding globulin is expected to be abnormal, like in obese individuals. Otherwise, total testosterone is sufficient for diagnosis and monitoring of treatment of hypogonadism. Testosterone levels may vary in the same individual at different test days; this may refer to biological or methodological factors or due to poorly defined reference ranges. The biological factors contributing to variation in circulating testosterone levels include genetic factors binding protein circadian production rhythm and seasonal variation. Therefore, guidelines have recommended 2 low testosterone tests to confirm the diagnosis of testosterone deficiency. The lectures also focused on testosterone deficiency in older people, where the studies have shown improvement in general well-being, correction of unexplained anemia, improvement in bone mineral density, and muscle strength. However, testosterone replacement in older people did not improve depression or cognitive function decline.

\section{Neuroendocrine Tumor Imaging}

Dr. Richard Auchus (USA) briefly introduced neuroendocrine tumors (NET). The morphology of these tumors contains dense secretory granules due to IHC, chromogranin, and synaptophysin. According to the mitotic 
index, they are graded in severity, i.e., low grade if the mitotic index is $<2 \%$, medium $2-10 \%$, and high grade if $>10 \%$. They are staged between 1 and 4 as per ENETs or AJCC criteria. For NET diagnosis, a biopsy is required, and comprehensive imaging modalities like ultrasonography, CT, MRI, 111InpPentetreotide, and 68Ga-DOTATAE. Endoscopic ultrasound is of great value specifically for pancreatic NET and is usually done by a skilled gastroenterologist.

MRI for localization of NET has to be done in the arterial phase to look for "Tumor Blush" as an example of liver metastasis in NET. Ga-Dotatate is very sensitive in picking metastatic disease for glucagonoma and insulinoma. Especially a $68 \mathrm{Ga}$ high-resolution scan with DOATATE in localization of primary insulinoma is more sensitive than an In-Pentetreotide-labeled nuclear scan. However, NET $<1 \mathrm{~cm}$ may not show in $68 \mathrm{Ga}$-Dotatate, and they require concordance by other imaging like MRI, $\mathrm{CT}$, and endoscopic ultrasonography. MIBG scan is specific for pheochromocytoma. The different molecules secrete from these NET used as tumor markers.

Regarding the treatment for low- to medium-grade tumors, surgery is the treatment of choice. For focal metastatic disease, surgery or radiofrequency ablation serve the purpose. For widespread metastasis, SSA is used. Everolimus and sunitinib are used as therapeutic agents, especially for pancreatic NET. Another unique modality of treatment is also implicated for these tumors by radionucleotide-labeled DOTATAE, e.g., 90Y-DOTATAE, 177Lu-DOTATAE for metastatic disease. Telotristat ethyl-TOH (tyrosine hydroxylase inhibitors) for serotonin excess in small and midgut NET is also used. For highgrade NET, chemotherapeutic agents and occasionally radiotherapy are also considered.

\section{Immunotherapy-Induced Endocrine Disease}

Dr. Carla Moran from Ireland elaborated on the evolving topic in endocrinology, i.e., immunotherapies induced endocrinopathies. Immune checkpoint inhibitors (ICI) are a new modality of chemotherapeutic agents used for various malignancies. They work through the activation of $\mathrm{T}$ cells and related pathways. These therapies are effective antineoplastic agents but result in different immune-mediated adverse effects on many organ systems, e.g., skin, lung, GI, and endocrine systems. Standard terminology Criteria classify these Immune-Related Adverse effects from immunotherapy for Adverse Events (CTCAE) in 5 grades according to the severity scales. Most endocrine-related immune-related adverse effects vary between grades 1 and 2, ranging from asymptom- atic patients to moderate symptoms. Anti-PD-1 agents are most commonly associated with endocrine disorders, followed by anti-CTLA- 4 and least by anti-PD-L1. The primary hypothyroidism is the most common endocrinopathy related with both anti- PD-1 and PD-L1 agents with a frequency of $2-13 \%$ and $9-12 \%$, respectively, followed by hyperthyroidism ( $1-7.8 \%$ and $<5 \%)$, hypophysitis $(0-1.9 \%$ and $<1 \%)$, and adrenal insufficiency $(<1 \%$ for both). Though subclinical hypo- and hyperthyroidism occur in an additional 13\% of patients, Dr. Moran also discussed screening and treatment algorithms for PD-1 and PD-L1 agents to avoid a delay in the diagnosis and management in such cases. She advised checking a baseline thyroid function test before initiating anti-PDI/ PDL-1 therapy, at least monthly for the first 6 months, followed by quarterly for the next 6-12 months and 6 months after that. In the case of an abnormal thyroid test suggestive of secondary hypothyroidism, the guideline suggests evaluating the pituitary profile for the picture of hyperthyroidism, suggested to treat as a usual case of thyroiditis by beta block NSAIDs, or steroid if painful, as Graves' disease is rare. Repeat thyroid function for 6 weeks; if persistent thyrotoxicosis, then do check, iodine uptake scan, and anti-TSI/TRAb antibodies.

Anti-CTLA-4 therapy was a cause of $0-17 \%$ of hypophysitis, followed by $0-7 \%$ cases of hypothyroidism and rarely Addison disease. Anterior hypophysitis is more common in males than in women, aged above 60 years, and suspects if there are symptoms of headache, fatigue, hyponatremia, anorexia, and nausea, rare to have visual field defects. It is recommended for these patients to have an MRI pituitary. The screening and management algorithm for anti-CTLA-4 therapy suggested checking baseline TSH, FT4, morning cortisol, and ACTH if cortisol is low. Check monthly for the first 6 months, followed by quarterly for the next 6-12 months and 6 months after that for TFT and annual for cortisol. Treatment is by the replacement for deficient hormone, and if MRI shows pituitary encroaching chiasma or severe headache, then a high dose of steroid, i.e., IV methylprednisolone and formal visual field assessment, should be taken.

ICI treatment needs to stop only in chiasm compression or development of severe site-threatening Graves' orbitopathy. In other instances, it is not advised to stop the therapy. Primary adrenal insufficiency and diabetes mellitus are rare occurrences with ICI therapy. Imaging the adrenal is mandatory to rule out metastasis; it shows inflamed adrenals and may have positive antiadrenal antibodies. There is a picture of T1D, and $70 \%$ of cases present with DKA, and 53\% have positive anti-islet $\mathrm{Ab}$ and 
mostly GAD Abs. Pancreatic imaging is not required. Diabetes is more common in susceptible HLA genotype DR4 (65\%) and usually requires lifelong insulin treatment.

\section{Diabetes}

What Is on the Horizon for Diabetes Management

Dr. Steven Kahn (USA) covered 2 crucial issues: (1) weight loss and glucose control with bariatric-metabolic surgery and medications, and (2) COVID-19 and diabetes: what lessons were learned so far and what lessons to be learned in the future.

Weight loss can be achieved to an equal extent by either bariatric-metabolic surgery or very strict lifestyle modifications of exercise and a very low-calorie diet. This will be associated with similar beneficial effects on the liver, muscle, adipose insulin sensitivity, insulin secretion, and glucose homeostasis. However, these were associated with contrasting gut biome changes, circulating bile acids, and branched-chain amino acid homeostasis. Furthermore, some patients may need medications in addition after bariatric surgery. Weight loss is associated with improved $\mathrm{HbAlc}$ and lipid profiles, but bone mineral density reduction also occurs. New data for drug therapy for obesity, including semaglutide treatment effect in people with obesity (STEP) development program, are exciting. Results produced positive weight loss in people with overweight and obesity (STEP1), diabetes, and obesity (STEP2), an adjunct to intensive behavioral therapy (STEP3). The cardiovascular safety study (SELECT) is ongoing, and its results are eagerly awaited. Data on Tirzepatide. A novel dual GIP and GLP1 RA are interesting. Phase III study (SURPASS 1) demonstrated impressive improvement in $\mathrm{HbA1c}$ and weight reduction.

Regarding COVID-19 and diabetes, he highlighted the increased hospitalization, need for intensive care, and mortality in diabetes. He also sifted through the COVID-19 link to diabetes by age and comorbidities. Glycemic status and the need for insulin do predict poor outcomes. He underscored a few words of caution regarding the emerging data on diabetes and COVID-19. (a) The poor quality of peer review, (b) most studies are retrospective, (c) a lot of "confounding by indication" was evident, (d) meta-analyses may count individuals more than once, and (e) there were few or small randomized clinical trials of the interventions. He commented on the phenomenon of "timeliness of recovery and solidarity in COVID-19 adaptive, pragmatic clinical trials. He quoted the conclusion that of the $>2,000$ planned drug studies examining COVID-19 treatment listed (www.covid-trials.org), most have delivered little or no directly useful information." Finally, concerning glucose-lowering agents in clinical trials in patients with COVID-19, he highlighted 2 ongoing studies that may be particularly relevant to clinical practice: (a) Dapagliflozin in respiratory failure in patients with COVID-19 (DARE-19) and (b) Semaglutide impact on myocardial injury in patients with COVID-19 (SIMPATICO).

\section{Immunotherapy in T1D: Can We Prevent Diabetes?}

Dr. Jay Skyler (Miami, USA) started by recognizing $\mathrm{T} 1 \mathrm{D}$ as an immunological disease. He also introduced the ideal therapeutic goals in T1d, namely preventing immune destruction, preserving B-cell mass or function, replacement or regeneration of B-cells, and automated insulin delivery. Next, he outlined the natural history of T1D from genetic predisposition (detected by genetic studies), to insulitis and beta-cell injury (mediated by cellular [T-cell] autoimmunity marked by the presence of various autoantibodies), to dysglycemia of prediabetes marked by loss of first-phase insulin response 9n IV GTT, and culminating into diabetes fulfilling the classical clinical and biochemical characteristics of T1D. He cited historical data supporting the immunological nature of T1D from finding from several workers and specifically from the T1D prevention studies. He also applied the types of prevention on the various stages of T1D. He took the audience through a smooth journey through all the T1D prevention trials and illustrated the different mechanisms targeted by various interventions. The discussion of the individual studies is beyond the scope of this report. However, he progressed, suggesting that the way forward is perhaps an aggressive combination strategy comprising 3 components of the immune system (innate immunity, adaptive immunity, and regulatory immunity) coupled with improving beta-cell health. He ended up illustrating this strategy by the Diabetes Islet Preservation Immune Treatment (DIPIT) trial using an anti-inflammatory agent such as an anti-TNF for immune modulation of ATG and anti-CD3, driving immune regulation (IL2), and preservation beta-cell heath using GLP-1-RA.

\section{Monogenic Forms of Diabetes}

V. Mohan (India) presented an excellent review on "monogenic" diabetes, i.e., diabetes that occurs due to the inheritance of a mutation in a single gene in contrast to other "polygenic forms"' of diabetes where several genes are implicated. Monogenic diabetes can be considered 2 
classes: genes involved in insulin secretion (such as neonatal diabetes and several forms of MODY) and those genes medication insulin resistance exemplified by lipodystrophy, type 1 insulin resistance syndrome, and leprechauns). He focused on the former group being more common. He illustrated the exact mechanisms causing the various forms. He elaborated on the definitions and precision medicine approach to several monogenic diabetes forms and related the genetic findings to the appropriate treatment, such as sulfonylureas in KCNJ11 and ABBC8 subtypes neonatal diabetes. He continued to elaborate on several examples. He also underscored the indications for genetic testing. He suggested that genetic testing should be undertaken in all patients suspected of T1D but have positive C-peptide and negative anti-GAD antibodies, all children with diabetes occurring before 6 months of age. Also, suspected early-onset T2D with no acanthosis nigricans and obesity described the merits and direct benefits. He concluded by 4 pearls of wisdom: (1) use both clinical information and nongenetic investigations to decide which patients need genetic testing; (2) good clinical phenotyping can make genetic testing costeffective; (3) genetic testing helps diagnose monogenic diabetes and defines the subtype, which helps with counseling, prognostication, and treatment; and finally, (4) genetic testing can also help change the therapy for some forms of diabetes, which can be life-transforming for patients and families.

\section{How Can We Limit the Development and Progression} of T2D in Youth and Middle Age?

Dr. Stephen Kahn addressed 3 themes in this presentation: first, the relative contribution of insulin sensitivity and beta-cell function in the control of glucose metabolism; second, the comparison of youth and adults and their respective response intervention; and third, an outlook on what may be next.

\section{A New Paradigm for the Treatment of T2D}

Dr. Anne Peters reflected on the ADA-EASD algorithm from selected angles. She stressed the critical role of lifestyle changes and that Metformin is the first choice. Following this, we need to ask questions about high-risk indicators, namely CKD or CVD. When these exist, there are compelling indications for SGLT2 inhibitors or GLP1 RA. Otherwise, the choice will depend on weight, cost, and risk of hypoglycemia. She reviewed several of the studies that led to these strategies. She summarized the CVOT data on all the classes. She elaborated on the SGLT2 Inhibitors and GLP1 RA mechanism of action, benefits, and side effects. She also discussed the data of various trials on SGLT2 inhibitors. So her take-home messages were to (1) target additional CVD risk reduction, (2) give options for therapy, (3) discuss nutrition, (4) watch for too much weight loss, and (5) consider $\mathrm{HbAlc}$ level in the context of individualized care.

\section{Thiazolidinediones: Due for a Resurrection or}

\section{Reincarnation?}

In clinical practice, Dr. Inzucchi attempted to address the potential for thiazolidinediones to answer his question. He reviewed the basics of insulin resistance and its relationship to cardiovascular risk by recalling the original work linking fasting hyperinsulinemia and metabolic syndrome to cardiovascular disease and reminded the audience of the early "common soil" hypothesis linking diabetes CVD. Next, he gave a brief history of thiazolidinediones and $\mathrm{CV}$ events and cited the benefits seen in the landmark PROactive trial. He also recalled the meta-analysis raising doubt about the efficacy and safety of rosiglitazone, and he drew a balanced view between the risks and benefits of pioglitazone as we stand in 2021. Next, he elaborated on the IRIS trial on valuable, robust data on both CV outcomes represented by RRR (ACS: 29\%; stroke: $25 \%$ ) and also DM prevention (RRR 52\% overall; most marked in IFG and the high HbA1c values) using the prediabetes data. He ended with providing some more insights into recent pioglitazone news (e.g., CV benefit in TOSCAIT study and benefit of pioglitazone on NASH data and the reassuring data regarding lack of risk bladder cancer). He also illustrated the place of pioglitazone in the algorithm of T2D management adopted by the ADA/ EASD and illustrated the comparative cost of various antidiabetic drugs.

Dr. Inzucchi concluded that: (1) insulin resistance is linked both epidemiologically and pathophysiologically to CVD. Therefore, it is logical to propose that an insulinsensitizing drug may have CV benefits, though challenging to demonstrate in randomized clinical trials. (2) In the IRIS trial, pioglitazone reduced the risk of stroke and MI in patients with insulin resistance and cerebrovascular disease, confirming the CV benefit suggested in the PROactive trial where type 2 diabetes with a macrovascular disease was studied. (3) Also, pioglitazone is the only glucose-lowering agent demonstrated to reduce atherosclerotic events directly. Although these effects are not necessarily linked, pioglitazone is also the only drug to prevent diabetes and improve ASCVD outcomes in the same trial. (4) Pioglitazone also appears to have a potential beneficial effect in NASH, including histological changes. There is, 
of course. Although the latter has been questioned, there are legitimate concerns about weight gain, edema, heart failure, bone fracture, and bladder cancer. Finally, (5) however, the role of this inexpensive generic drug needs reassessment in the treatment of patients with T2D, especially those with CVD and perhaps in those with insulin resistance or prediabetes with cardiovascular disease.

\section{Can the Prevention of Diabetes Lead to the Prevention} of Cardiovascular Disease?

Dr. Silvio Insucchi (USA) addressed the above question by providing background arguments for the link between diabetes and cardiovascular disease based on the mega trials of hyperglycemic control (DCCT, UKPDS, ACCORD, VADT, and ADVANCE). He further reviewed lifestyle-based and pharmacological studies on the prevention of diabetes (Da Qing, Finish DPS, and the USA DPP). He further analyzed CVD prevention data from the diabetes trials to address whether we can prevent CVD by preventing or delaying diabetes? From the evidence he presented, he came to the following conclusions: First, due to increasing rates worldwide, there is a need for effective and safe interventions to prevent T2D. Second, more than a dozen large diabetes prevention randomized controlled trials have demonstrated a relative risk reduction of $32-58 \%$ with lifestyle changes and 19 $78 \%$ with certain glucose-lowering and weight loss medication. He questioned whether drugs prevent or mere delay that you have read this remains controversial. Third, while logical, evidence that preventing T2D reduces $\mathrm{CVD}$ is scant (low-risk patients or short duration of follow-up). Also, there are suggestions of a long-term benefit in follow-up studies of lifestyle intervention trials. Fourthly, 2 antidiabetic medications, pioglitazone, and dapagliflozin have been assessed for new-onset diabetes due to the large CV outcome trials (IRIS and DAPA-HF), both involving high CV risk patients (stroke and HFrEF). In these trials, participants randomized to active therapy developed new-onset diabetes less frequently than those randomized to placebo (RRRs: 52; 32\%). Finally, so far, it is not yet known to what degree (if any) diabetes prevention might be linked to.

\section{Technology in Diabetes Care}

Use of Devices in People with Diabetes and TIR

versus $\mathrm{HbA1c}$

Both Drs. Anne Peters and Jay Skyler addressed these issues from slightly different perspectives. However, the presentations were complementary rather than contradictory. Dr. Peters started by discussing the limited infor- mation provided by $\mathrm{HbA} 1 \mathrm{c}$ in the context of patients with T1D with high variability. This stresses the value of CGM in clinical practice. She reviewed the attributes of various available sensors produced by different manufacturers. Alerts for high and low values are very valuable. She described the concept of AGP and how these need to be interpreted. Time in the range is essential; "glucose management indicator" is the new name for the "estimated HbA1c." Examination of the daily pattern can be beneficial too. Various insulin delivery systems are now available, from conventional to automated systems combining pumping systems and sensors and hybrid closed-loop systems.

Dr. Skyler started by using the T1D exchange data to illustrate the more significant impact of CGM use than insulin delivery methods (MDI vs. Pump) on the HbAlc level. He reiterated what was stated by Dr. Peters on the limited value of $\mathrm{HbAlc}$ versus time in range (TIR) and measures of glucose variability. He also added that the glucose management index bypasses the limitations of measured $\mathrm{HbAlc}$, such as anemia and uremia. He described the standardization of CGM metrics and the interpretation of 10 core metrics. He elaborated on the TIR being $>70 \%$ between 70 and 180 (i.e., $>16 \mathrm{~h} 48 \mathrm{~min}$ ). He illustrated the interpretation of various metrics in the real world and used actual patient data. He also alluded to the differences between HbAlc and GIM. He also demonstrated that similar to HbA1c, TIR is related to the frequency of complications (retinopathy, measures of peripheral nerve function, and intima-media thickness).

A Century of Diabetes Technology: Signals, Models, and Artificial Pancreas Control

Boris Kovatchev (USA) elaborated on the subject by (1) reviewing the history of diabetes devices, (2) examining the signals and insulin delivery mechanisms available to the contemporary treatment of diabetes, (3) summarizing the mathematical models and in silico preclinical trials enabling diabetes research, and (4) reviewing the latest in automatic control development-the artificial pancreas. In conclusion, in the 100 years since the discovery of insulin, an ecosystem of devices has evolved dedicated to the treatment of diabetes. Generally, these devices fall into 2 categories: those that generate relevant signals (e.g., glucose or other physiological readings) and those that actuate the delivery of insulin or other medications. Mathematical models and in silico preclinical trials enable diabetes research and the development of automated closed-loop control of diabetes, known as the "artificial pancreas." The speaker closed by stating that "To-
Beshyah/Rashid/Ibrahim Abdelgadir 
day, the 'artificial pancreas is coming of age with 2 systems already on the market, approved for use by children and adults.”

Diabetes Data Science: The Virtual Image of the Patient Approach to Personalized Diabetes Medicine

Boris Kovatchev (USA) presented a very thought-provoking lecture on diabetes data science. He addressed 4 themes: (a) introducing advanced data science solutions to the analysis and interpretation of CGM data. (b) examining the classification of CGM daily profiles into identifiable clusters. (c) linking sequences of daily CGM profiles into individual glycemic trajectories for each patient and (d) defining the virtual image of the patient and previewing its use for personalized treatment optimization.

Glucose Variability: Timing, Risk Analysis, and

Relationship to Hypoglycemia in Diabetes

Boris Kovatchev (USA) addressed these issues by covering 4 themes: (1) the optimization problem in diabetes, (2) risk analysis of glucose data, (3) assessment and prediction of the risk for hypoglycemia, and (4) reconciling $\mathrm{HbA1c}$ and TIR metrics. He started to formulate the treatment of diabetes as a quantitative optimization problem whereby maintaining the amount of glucose dissolved in the whole blood is maintained constant. $\mathrm{He}$ quoted the definition coined by Dr. Cryer's doctrine that "The glycemic goals in diabetes represent a trade-off between glycemic control (optimal HbAlc) and iatrogenic hypoglycemia." The way to achieve this trade-off is minimizing the variance in blood glucose. Current consensus uses TIR at around $70 \%$ of the time with stringent criteria in some circumstances such as pregnancy. Metrics of glycemic control vary according to the data source. For instance, $\mathrm{HbA1c}$ has a quick resolution in months, whereas episodic monitoring using SMBG and flash glucose monitoring has a temporary resolution in days and weeks, and finally, GCMS is based on real-time measurements, which has a temporary resolution in minutes. However, various metrics have been introduced for each one to achieve good glycemic control. Second, he built a risk analysis framework to offer mathematical solutions assisting the clinical optimization of diabetes treatment. Third, he reviewed examples of prediction of hypoglycemia, severe hypoglycemia, and extreme hyperglycemia, and fourth he attempted to reconcile the long-standing perceived discrepancy between $\mathrm{HbA} 1 \mathrm{c}$ and TIR using a new modeling technique.

He concluded that (1) to transition from art to science to occur, the treatment of diabetes needs to be formulated as a quantitative optimization problem, (2) a recent international consensus proposed metrics and graphs describing the state of a person's diabetes in quantitative terms. TIR (70-180 mg/dL) was suggested as a primary indicator of diabetes control. The risk analysis framework reviewed in his lecture is specifically designed to assess and predict the risk for extreme events, i.e., hypoglycemia, severe hypoglycemia, and extreme hyperglycemia. Furthermore, Finally, HbA1c and TIR reflect the same underlying process of glycemic control. However, a well-established discrepancy between their values can be reconciled using a new modeling technique.

\section{Neuropathy and Diabetic Foot}

Diagnosis and Treatment of DSPN in Clinical

Practice

Dr. Dan Ziegler (Germany) opened by highlighting the epidemiological facts about diabetic sensorimotor polyneuropathy (DSPN). He recalled the simple definition for clinical practice stated in the ADA Position Statement (2017), namely the presence of symptoms and/or signs of peripheral nerve dysfunction in people with diabetes after excluding causes. However, up to $50 \%$ of diabetic peripheral neuropathies may be asymptomatic, whereas neuropathic pain is present in up to $25 \%$ of individuals with diabetes. He stressed the fact that the DSPN remains significantly undiagnosed and untreated. Neuropathy can predict the risk of cardiovascular disease. More recently, nerve dysfunction has been recognized in individuals with prediabetes and highlighted the link of neuropathy to severe insulin deficiency phenotypes of type 2 diabetes.

Prof. Zeigler analyzed the relationship between the types of fibers and various clinical findings and modalities of clinical evaluation and analyzed the time course of different symptoms from the development of diabetes onwards. He then reiterated the evidence-based recommended timing and frequency of monitoring for neuropathy. He also described the optimal methods for screening by history and physical examination using basic tools such as monofilaments and tuning fork. He strongly recommended using the Michigan Neuropathy Screening Instrument to score to quantify the risk using a cutoff point $>2$. He next introduced newer devices and methods for DSPN assessment. Quantitative sensory testing tools include electronic tuning fork ETF128, VibraTip, and nerve check. Sural sensory nerve conduction velocity can be used. Also, testing of the sudomotor function can be evaluated using a simple Neuropad and more advanced Sudoscan. Nerve conduction studies for large fibers and 
thermal thresholds have their advantages and disadvantages discussed in detail. The latest methods include skin biopsy with immunohistochemistry studies and corneal confocal microscopy, which may not be widely available and are primarily used in research. Exclusion of other causes is essential using standard tests.

Management of DSPN includes diabetic (glycemia) control, symptomatic treatment, and pathogenetic therapies. Regeneration of corneal nerves has been shown after normalization of blood glucose by transplantation in T1D, but this has not been confirmed in T2D trials. Hence, ADA recommends a preventative approach. Preventative measures include optimizing glucose control as early as possible to prevent or delay the development of DSPN and cardiovascular autonomic neuropathy in people with diabetes and to slow the progression of DSPN in T2D. The multifactorial approach includes addressing risk factors for people with diabetes and lifestyle interventions in prediabetes and T2D. He alluded to the treatment-induced neuropathy associated with aggressively reduced blood glucose over a short period. However, this recovers spontaneously within a year.

He elaborated on the pathogenesis of neuropathy to base his therapeutic approaches such as thioacetic acid, benfotiamine, and methylcobalamin. He reviewed the various trials that provided supporting data.

Regarding treatment effects in neuropathic pain, Dr. Xeigler started by listing the required assessments for chronic pain treatments, namely pain intensity, relief, mood, functionality, and sleep. He recommended a consistent use of numerical rating scales for pain intensity $(0$ $=$ no pain; $10=$ intolerable, with $4+$ being usually an indication for treatment). Drugs target various sites of action spreading from the periphery to the cortex. He presented a meta-analysis of pharmacotherapies painful neuropathy, including opioids and anticonvulsants. Sodium channel blockers, alpha-lipoic, and anticonvulsants were expressed as $>30$ or $>50$ pain reduction, and he illustrated several individual studies. Combination therapy can include symptomatic agents or symptomatic with pathogenetic agents.

On an individual basis, therapeutic agents need to be chosen by considering the comorbidities such as depression, generalized anxiety disorder, coronary artery disease, and autonomic neuropathy. Last but not least, a large array of nonpharmacological options is also available and need to be considered.

He left the audience with a "DSPN treatment algorithm" that progresses from assessment of the patients and diabetes to evaluation of the nature of presenting morbidity (asymptomatic DSPN, symptomatic nonpainful DSPN, and painful DSPN), leading to making a rational choice of pathogenic therapy for the first, use of analgesics for the third, and perhaps a combination for the middle. He suggested the order for the use of pharmacotherapy in 3 groups as follows: first: duloxetine, amitriptyline, pregabalin, and gabapentin; second: capsaicin $8 \%$ patch and tramadol; and third: potent opioids and sodium channel blockers. With considerations of the use of combinations and/or use of nonpharmacological treatment, refractory cases should be referred to specialized centers. His take-home messages included: (1) polyneuropathy is an early rather than late diabetic complication prevalent in prediabetes. (2) Protective effects of near normoglycemia on nerve function are proven in T1D but not T2D. (3) Symptomatic analgesic monotherapy only relieves the pain without targeting the underlying neuropathy and it has limited efficacy. (4) Multimodal treatment of DSPN should consider the individual risk profile, pathogenetic treatment, and pain management using pharmacotherapeutic agents in isolation initially or in combination when needed as well as nonpharmacological options.

\section{Management of Diabetic Foot: What Does Success}

Look Like?

Mark Tagoe (UK) gave a "reflective" presentation on facts of diabetic foot disease globally and rather than address the original questions: How are we doing regionally? He highlighted the burden of diabetes and diabetic foot disease using data from the UK to illustrate his arguments. Moving to prevent diabetic foot disease, he considered the factors leading to ulceration and amputation under 3 categories; intrinsic, extrinsic, and behavioral. The intrinsic factors include callous formation, Charcot's joints, foot deformity, and limited joint mobility. On the other hand, extrinsic factors comprise footwear, foreign body, trauma (thermal and mechanical), and patients' knowledge about risk factors. Behavioral factors involve barefoot walking, smoking, lack of regular examination of foot and footwear, and poor hygiene. He recalled the pillars of good diabetes care for people admitted to hospital with "diabetic foot" involving multidisciplinary team (MDT), being under a named consultant within the MDT, robust protocols, and pathways with regular review of care and presence of foot protection program. He described the recommended 3-level model of care network comprising basic, intermediate, and centers of excellence type of care. He introduced the concept of "diabetic foot attack" in its typic or infected type and the atyp-
Beshyah/Rashid/Ibrahim Abdelgadir 
ical forms such as the ischemic and Charcot joint. He highlighted some (good and bad) special features in the Middle East and Arab countries that may affect diabetes and used UAE data to illustrate various arguments. $\mathrm{He}$ used several clinical cases to illustrate the various vascular, tissue damage, and mechanical challenges. He also took time to illustrate the value and optimal provision of diabetic footwear. He underscored the reportedly varying availability and composition of diabetic foot MDT. He also used the teams in Dubai to illustrate the concept of MDT.

In summary, the speaker stressed that the problem of diabetic foot is universal. Patient education and motivation are needed to prevent diabetic foot problems, particularly in the wake of rising numbers of people with diabetes. He closed by stressing the essence of good care and the value of formal MDT. He left it to individual professionals and groups to see where they stand from this.

\section{Metabolism}

\section{Obesity}

Counseling on the Fundamentals of Lifestyle Change for Obesity: Diet, Physical Activity, and Behavior

Dr. Robert Kushner (USA) divided his presentation into 3 themes: (a) role of diet in obesity management, (b) role of physical activity in obesity management, and (c) behavioral counseling. The essential components of an effective obesity management program are physical activity, behavioral modification, and diet. Advanced components comprise antiobesity medications (AOM) and bariatric/metabolic surgery. He linked the drivers of behavior (namely environment, culture, biology, and psychology) to diet and physical activity as the 2 components of behavior. He then underscored the fundamental guiding principles for dietary counseling: (1) creation of energy deficit and (2) prescribed diet based on patient preferences and health status. He illustrated several of the multiple dietary patterns "diets" such as Mediterranean diet, DASH food pyramid, USDA MyPlate, nordic diet, lacto-ovo-vegetarian, and low carbohydrate diet. However, he illustrated that (1) calorie restriction is primarily responsible for weight loss rather than the macronutritional components and adherence to the prescribed diet. He next alluded to the concepts, "meal replacement" strategy for weight reduction and diabetes remission data from the DIRECT study, and discussed the relationship of ultra-processed food to mortality.

EDEC 2021 Highlights
Dr. Kushner then turned to physical activity and introduced the concept of "movement portfolio." He recognized 3 levels of movement, namely: exercise (recreation by aerobic or strength training), activities of daily living (work, school, household, and transportation), and sedentary behavior (physical inactivity). He recalled the cardioprotective mechanisms of physical activity such as psychological, antiarrhythmic, antithrombotic, antiatherosclerotic, and hemodynamic effects. He discussed the limited role of physical activity in weight reduction unless it is accompanied by calorie restriction, and he contested the relative contributions of diet and exercise, given amounts of weight loss.

In the last part, Dr. Kushner elaborated on the principles of behavior change. He started by defining ambivalence and how behavior change balances motivation (the despite) and resistance (impediments to change). A physician needs to find the leverage point to facilitate the behavior change. He described the most common behavioral theories used in practice. Relevant ones include social cognitive theory/ecological models, cognitive, motivational interviewing, stages of change model, theory of planned behavior, health belief model, and self-determination. There are several determinants of making a difference in behavior change. For instance, knowledge is necessary but insufficient to make a health change. Also, effective interventions combine education with behaviorally oriented counseling to help patients acquire the skills, motivation, and support needed to alter the targeted behavior. However, first patients need to be helped understand why they behave the way they do. This is done by asking the right questions and listening. Next, he discussed the transtheoretical model stage of change, namely precontemplation, contemplation, preparation, action, and maintenance. He also demonstrated the health belief model, where a reasonable sum is needed between a perceived threat (susceptibility and seriousness) and outcome expectation (perceived benefits and barriers) together with self-efficacy (i.e., perceived ability to carry out the recommended action). Cognitive-behavioral therapy is a well-established methodology. Simply monitoring often changes behavior. Self-monitoring in this context, regular weight checking at home, monitoring of $\mathrm{BP}$, and self-monitoring of blood glucose are familiar examples. Similarly, several electronic tracking and interactive weights for weight management are available.

Finally, he highlighted the 5 steps to facilitate a behavior change: (1) identification of the behavior change goal, (2) review when, where, and how behaviors will be performed, (3) record the patient's behavior change, (4) re-

Dubai Diabetes Endocrinol J 2022;28:1-19 15 
view progress at the next treatment visit, and (5) congratulate the patient on success and not criticize shortcomings. Goals should be SMART (specific, measurable, attainable, realistic, and timely). Changes need to be made into habits depending, planning, and accountability by building habits that stick. He ended by presenting a comprehensive program to achieve $7-10 \%$ weight loss in 1 year or more.

In conclusion, lifestyle modification remains the foundation of obesity management. As long as the diet is calorie-reduced, patients can follow a diet that they prefer. Also, the movement portfolio needs to be remembered for physical activity, including increased ADL and exercise and reduced sedentary time, and finally, the development of behavioral skills.

Pharmacotherapy for Obesity: Understanding the Appetite-Behavior Connection

Dr. Kushner illustrated the complex mechanisms regulating food intake by interacting internal biological factors with the external environment. Two CNS pathways play a crucial role in regulating eating habits, cravings, and weight, namely the hunger satiety/system and the reward system. Antiobesity drugs suppress appetite by their actions on various components of the 2 systems. Appetite has several components, and understanding these helps physicians and patients understand how they are losing weight. Currently, available antibody medications belong to various classes that exert their effects by appetite regulation, such as liraglutide, phentermine/topiramate, naltrexone/bupropion, or fat reduction absorption orlistat. Dr. Kushner stressed that the primary purpose of adjunctive medications in obesity is to help patients adhere to a low-calorie diet more consistently to achieve sufficient weight loss and health improvement when combined with increased physical activity. He reviewed the effects of some of the antiobesity drugs on various components of eating behavior. He also explained how specific effects decrease, but weight loss is maintained.

The speaker concluded that (1) lifestyle modification is the foundation of obesity management. Weight loss occurs by achieving a negative energy balance, (2) Antibody medications biochemically alter appetite regulation, including reducing hunger and food cue reactivity and increasing satiety. (3) Despite weight loss maintenance, perception of hunger and fullness return toward baseline.

Bariatric Surgery: Devil or Divine?

In his third presentation, Dr. Kushner attempted to answer several questions: first, describe the surgical pro- cedures and mechanism of action; second, define complications, management, and beneficial outcomes; thirdly, discuss the effects on diabetes; and lastly, discuss the occurrence of weight regain and its management. He discussed the clinical assessments to identify the most important driving factors for weight gain (medications, psychological, and medical).

Dr. Kushner reminded me that the most familiar bariatric surgery in the USA is the sleeve gastric surgery, followed by RYGB bypass, and the less used one is the biliopancreatic diversion, which is the most malabsorptive procedure. In weight reduction, different mechanisms of bypass surgeries include less mechanical space for food, a significant increase in gut hormones like GLP-1 and GIP (satiety hormones) and PYY, and less ghrelin (hunger) hormones of microbiota fat metabolism, as well as improvement in insulin resistance. Bariatric surgery significantly reduces the ghrelin hormone compared to a mild increment in the lifestyle modality of weight reduction.

Data from the LAB study followed 2,500 patients with bariatric surgery for 7 years. An average 35\% (15-45\%) body weight loss was observed and maintained for around 7 years, a level of reduction that has not been shown using lifestyle or medical management. The best time to look for the maximum benefit of the surgery was between 12 and 18 months. Other benefits from the Swedish Obesity Study included overall mortality reduction by $30 \%$, in addition to improvement or remission (within 2 years) of diabetes, hypertension, dyslipidemia, and sleep apnea. Complications of bariatric surgery include heartburn, GERD, vitamins and nutritional deficiencies, weight regains, and the more severe complications, including mortality and surgical complications, which are pretty uncommon.

Dr. Kushner also alluded to the weight regain after bariatric surgery and presented the data from LAB and Swedish Obesity Study, which showed many patients regaining around $25 \%$ of the lost weight. This is to be prevented by reemphasizing the lifestyle measure, adding weight-reducing medication, and probably resurgery if needed. However, the level of evidence for the last recommendations is low. The speaker reviewed the benefits of AOM, showed that the average weight reduction with the AOM is between 6 and 12\%, and pointed to the availability of various medications. As of now, we do not have many approved medications for obesity.

His conclusions were as follows: (1) metabolic and bariatric surgery is an effective and acceptable treatment for patients with severe obesity or moderate obesity with comorbid conditions. (2) Demonstrable clinical benefits
16

Dubai Diabetes Endocrinol J 2022;28:1-19 DOI: $10.1159 / 000520783$
Beshyah/Rashid/Ibrahim Abdelgadir 
are seen in multiple obesity-related conditions and improvement in mortality. (3) However, some weight regain is an expected occurrence associated with the loss of health benefits, most notably T2D. Vitamin and nutritional deficiencies, such as iron, vitamin D, B12, and other micronutrients, occur. (4) Lifelong vigilance and management of patients with obesity who had bariatric surgery are needed to address complications and nutritional deficiencies and be alert for weight regain.

\section{Present and Future Lipid-Modifying Therapy}

Patrick Moriarty, USA, reviewed the role of the nonstatin lipid-lowering therapy. He started with an overview of the genetic and biochemical basis for regulating lipid homeostasis in health and disease and the role of apoB lipoprotein and LDL receptor. He highlighted the role of hyperlipidemia in the etiology of cardiovascular disease and the role of reducing lipids. He further presented lipid-modifying therapy (LMT) for the treatment of apoBcontaining lipoproteins (i.e., LDL, VLDL, chylomicrons, and $L p[a])$. He recalled the history, mechanism of action, and lipid-lowering response of the current classes of LMT, including statins, bile acid sequestrants, cholesterol absorption inhibitors, fibrates, nicotinic acid, PCSK9 inhibitors, and omega 3 and lipid aphesis.

He then progressed to address newer LMT and finished by reviewing potential future LMT to treat apoBcontaining lipoproteins. Reduction of cholesterol synthesis by inhibiting ACL 2 steps upstream from HMG-Co A reductase. Bempedoic acid drives down LDL cholesterol by increasing LDL-receptor expression. Bempedoic acid is approved and has the advantage of not entering muscles, making it suitable for statin-intolerant patients. $\mathrm{He}$ presented Phase III data on this class with and without combination with ezetimibe with impressive results. Next, he described the role of APO-C III and angiopoietin-like protein in triglycerides and LDL-C metabolism. Since RNA-targeted antisense drugs block the translation of a specific protein, these antisense drugs can be produced to target and give translations. He alluded to the newly approved volanesorsen that lowers triglycerides by $70 \%$ in the APPROACH trial and significantly reduces the risk of pancreatitis. Another drug, evolocumab, lowered LDL-C by about 50\% in homozygous FH. Another injectable drug (Inclisiran) lowers LDL by $50 \%$ in resistant hyperlipidemias in the study ORION 10 and 11 . He finished by discussing the impact of raised $L p(a)$ and the limited effects of various medications. However, lipid apheresis has recently been shown to reduce it and cardiovascular events. He also alluded to future promising drugs of effects on various pathways with a remarkable reduction in LDL and should be available soon.

The speaker concluded that: (a) apoB lipoprotein is the primary source of atherosclerosis and cardiovascular disease; (b) the reduction of apoB has demonstrated a lowering of CVD risk; (c) lipid apheresis reduces apoB-containing lipoproteins and is approved for lowering LDL-C and $L p(a)$; and finally, (d) newer therapies will potentially further reduce apoB-containing lipoproteins.

\section{Nonalcoholic Steatohepatitis: The Overlooked Complication of Diabetes}

Kenneth Cusi (USA) started by defining the different entities, the various conditions and the sequence of events, and the factors driving the progression, from fatty liver to NAFLD, NASH, and leading to the risk of cirrhosis. He also stressed the bidirectional relationship between T2D and NAFLD and the negative impact on the other.

Diagnosis can be established by calculating the FIB-4 score (preferred than the NFS) and elastography (fibroscan). Despite the recognized high prevalence of NAFLD in people with T2D, there is no systematic screening. However, regarding management, antifibrotic drugs have failed. Therefore, the management strategies moved upstream to anti-inflammation. In people with diabetes, management entails optimizing glycemic control, weight loss, pioglitazone, and possibly vitamin E and semaglutide. Lower doses (15 mg) are associated with fewer side effects than higher doses $(30 \mathrm{mg})$. They may be better when combined with SGLT2 inhibitors and semaglutide. These should be given more preference in the management of diabetes and NASH.

His take-home message included: (1) NAFLD is common in primary care and endocrinology clinics in patients with T2D. (2) It is associated with increased morbidity and mortality. (3) There is still significant clinical inertia and diagnostic a; algorithms combining blood tests and imaging, and (4) diagnosis today is vital as some interventions can help patients. Pioglitazone is the Metformin for NASH; semaglutide and SGLT2 inhibitors are possible players. There are new agents in development.

\section{Diabetes and Endocrine Challenges in the COVID-19 \\ Pandemic \\ COVID-19 and Diabetes Mellitus}

Manel Puig-Domingo (Spain) gave a quick overview of the epidemiology, clinical aspects, and management of the interplay between COVID and diabetes. He emphasized a greater prevalence of diabetes in hospitalized ver- 
sus no hospitalized COVID-19 patients. This reflects the burden of severity partly because the inflammasome response to COVID-19 is amplified in people with diabetes. Diabetes is present in 1:3 patients with COVID-10 who are admitted to intensive care units, requiring mechanical ventilation, and those who are dying. Furthermore, mortality in COVID-19 infection is age-dependent and morbidity-dependent with glycemia and glycemic variabilitydependent, and diabesity confers a 3-5 increased risk of mortality. Finally, timely and adequate insulin therapy and nutritional support are recognized to decrease mortality in people with diabetes infected with COVID-19.

COVID-19 and Endocrine Disease: A Focus on Bone Health

Andrea Giustina (Italy) was invited to address the link between COD-19 and endocrine conditions. However, he chose to focus on bone health and calcium homeostasis. Nonetheless, he started with a short introduction to the endocrine glands/organs affected by COVID-19, sick euthyroid syndrome, possible transient hypothalamic-pituitary dysfunction, hyponatremia, probable higher susceptibility in adrenal insufficiency, and Cushing's syndrome. They are worse outcomes in obesity and diabetes. Low vitamin $\mathrm{D}$ deficiency is associated with worse outcomes. Obesity affects the outcome through the decreased respiratory reserve, comorbidities, increased viral shedding, and chronic inflammations. In the remainder of his presentation, Dr. Giustina discussed the issues of hypocalcemia, vitamin D deficiency, and antiosteoporotic therapy during COVID.

Dr. Giustina noted that hypocalcemia is an emerging widespread laboratory finding in COVID-19 with substantial clinical implications. As previously demonstrated for other viruses, calcium could also play a central role in viral infections and replicative mechanisms of SARSCoV-2. By predicting hospitalization and poor outcome in COVID-19 patients, hypocalcemia could have a role as a marker of disease aggressiveness. Hypocalcemia would be a valuable and easy to measure laboratory parameter to identify severe cases in the emergency department for clinicians. Furthermore, hypocalcemia per se, when severe, can complicate the cardiovascular and neurological picture of COVID-19. Finally, he underscored that the above observations suggest that calcium monitoring and adequate supplementation are indicated in all hospitalized patients with COVID-19 infection, particularly in even subclinical hypoparathyroidism.

Regarding vitamin D and COVID, he pointed out that mechanistic insights and available findings, albeit limited and nonunivocal, suggest a possible role of vitamin D status in the risk of COVID-19 infection and severity. Large randomized clinical trials of interventions to reduce vitamin $\mathrm{D}$ deficiency are needed to determine if those interventions could decrease COVID-19 incidence and clinical impact. Furthermore, trials should include broad population interventions and interventions among groups with increased risk of vitamin D deficiency and/or COVID-19 with possibly hard clinical endpoints.

Concerning the issues of VF and COVID-19, Dr. Giustina highlighted several observations of mechanistic and practice values, for instance, pathophysiologically. VF could be hypothesized to be linked to increased combined cardiorespiratory risk since they may be associated with cardiovascular comorbidities and predispose to respiratory failure and infection. Clinically, the results of their study demonstrate that VFs are one of the most frequent comorbidities in severe COVID-19 infection. VF almost reached the level of one of the few clinical features which has been consistently shown to be strongly correlated with COVID-19 risk and prognosis, such as arterial hypertension with which VFs correlate well and with a much higher prevalence than the other frequently reported COVID-19 comorbidities such as coronary artery disease, diabetes mellitus, and chronic kidney disease. Furthermore, VFs were clinically meaningful associated with a doubling in mortality risk, significantly higher in patients with severe compared to mild and moderate FVs.

The speaker also commented that it was interesting to note that their data support a hypothesis alerting the medical profession regarding the high likelihood of an increased risk of skeletal fragility and osteoporotic fracture with potential grave consequences on overall health status and chances of recovery of affected patients. These data also reinforce the need to implement the published recommendations concerning the importance of bone fragility care during the COVID pandemic, particularly those already on treatment, as well as continuity of care, monitoring of bone density, and initiation of antiresorptive therapy during hospitalization.

\section{Conclusions}

The virtual EDEC 2021 provided a comprehensive review of topical issues concerning clinical practice and research in diabetes, endocrinology, and metabolism. Predictably, the thyroid took the scene's center, spanning many subjects from hypothyroidism, pregnancy care,
18

Dubai Diabetes Endocrinol J 2022;28:1-19 DOI: $10.1159 / 000520783$
Beshyah/Rashid/Ibrahim Abdelgadir 
thyroid nodules, and cancer management. Rational approaches to pituitary disease and acromegaly were valuable for practicing endocrinologists. Recent advances in adrenal disease were precious, practical, and futuristic. The reviews on bone metabolism and calcium homeostasis at large and during the COVID-19 pandemic were exciting. On the diabetes side, emphasis on the cardiovascular outcomes, use of newer agents when compelling indications exist, and the role of technology were precious contributions. Some of the classical questions were addressed and revisited, such as endocrinology of thalassemia, precocious puberty, and testosterone replacement. The newer and future LMTs were stimulating mainly when high-risk patients were considered. Many new concepts were introduced in diabetes care and endocrinology, bringing the audience to the forefront of research and world-class clinical practice.

\section{Acknowledgments}

The authors are grateful to the Emirates Diabetes Society and the EDEC-2021 logistics organizer (MCI) for allowing extended online access to the recorded proceeding.

\section{Statement of Ethics}

Ethical approval is not required for a conference abstract study.

\section{Conflict of Interest Statement}

There are no conflicts of interest. The authors presented their perceptions of the proceedings of the congress. The speakers cannot be held responsible for any misrepresentation. The contents do not reflect the position, views, or opinions of their employers or their affiliations.

\section{Funding Sources}

There were no funding sources.

\section{Author Contributions}

All authors contributed equally to the drafting, development, and finalization of the manuscript.

\section{Data Availability Statement}

The data (Conference Abstracts) supporting this paper are published in the same Journal Issue/Supplement.
References

\footnotetext{
1 Proceedings of Abstracts 10th Emirates Diabetes and Endocrine Congress, February 27-29, 2020, Dubai, UAE, 27. Dubai Diabetes Endocrinol J; 2021.p. 18-36. doi 10.1159/000514044.

2 2021, Dubai, UAE. Dubai Diabetes Endocrinol J. 27:71-113.
} 